\title{
Comparative analysis of spreading depolarizations in brain slices exposed to osmotic or metabolic stress
}

\author{
Rita Frank, Ferenc Bari, Ákos Menyhárt ${ }^{* \dagger}$ and Eszter Farkas ${ }^{* \dagger}$
}

\begin{abstract}
Background: Recurrent spreading depolarizations (SDs) occur in stroke and traumatic brain injury and are considered as a hallmark of injury progression. The complexity of conditions associated with SD in the living brain encouraged researchers to study SD in live brain slice preparations, yet methodological differences among laboratories complicate integrative data interpretation. Here we provide a comparative evaluation of SD evolution in live brain slices, in response to selected SD triggers and in various media, under otherwise standardized experimental conditions.

Methods: Rat live coronal brain slices $(350 \mu \mathrm{m})$ were prepared $(\mathrm{n}=51)$. Hypo-osmotic medium $\left(\mathrm{Na}^{+}\right.$content reduced from 130 to $60 \mathrm{mM}, \mathrm{HM}$ ) or oxygen-glucose deprivation (OGD) were applied to cause osmotic or ischemic challenge. Brain slices superfused with artificial cerebrospinal fluid (aCSF) served as control. SDs were evoked in the control condition with pressure injection of $\mathrm{KCl}$ or electric stimulation. Local field potential (LFP) was recorded via an intracortical glass capillary electrode, or intrinsic optical signal imaging was conducted at white light illumination to characterize SDs. TTC and hematoxylin-eosin staining were used to assess tissue damage.
\end{abstract}

Results: Severe osmotic stress or OGD provoked a spontaneous SD. In contrast with SDs triggered in aCSF, these spontaneous depolarizations were characterized by incomplete repolarization and prolonged duration. Further, cortical SDs under HM or OGD propagated over the entire cortex and occassionally invaded the striatum, while SDs in aCSF covered a significantly smaller cortical area before coming to a halt, and never spread to the striatum. SDs in HM displayed the greatest amplitude and the most rapid propagation velocity. Finally, spontaneous SD in HM and especially under OGD was followed by tissue injury.

Conclusions: While the failure of $\mathrm{Na}^{+} / \mathrm{K}^{+}$ATP-ase is thought to impair tissue recovery from OGD-related SD, the tissue swelling-related hyper excitability and the exhaustion of astrocyte buffering capacity are suggested to promote SD evolution under osmotic stress. In contrast with OGD, SD propagating under hypo-osmotic condition is not terminal, yet it is associated with irreversible tissue injury. Further investigation is required to understand the mechanistic similarities or differences between the evolution of SDs spontaneously occurring in HM and under OGD.

Keywords: Brain slice, Cerebral ischemia, Spreading depolarization, Osmotic stress, Oxygen-glucose deprivation

\footnotetext{
*Correspondence: menyhart.akos@med.u-szeged.hu; farkas.eszter.1@med.uszeged.hu

${ }^{\dagger}$ Ákos Menyhárt and Eszter Farkas-Co-senior authors

Department of Medical Physics and Informatics, Faculty of Medicine

and Faculty of Science and Informatics, University of Szeged, Korányi fasor

9, 6720 Szeged, Hungary
}

\begin{abstract}
Background
In traumatic brain injury, stroke and anoxic brain injury caused by cardiac arrest, spreading depolarization (SD) plays a central pathophysiological role [1]. SD is also implicated in specific chronic neurological disorders including migraine with aura [2] or sudden unexpected
\end{abstract}


death in epilepsy (SUDEP) [3]. SD sets off in the cerebral gray matter from a focal, critical tissue volume depolarizing synchronously $\left(\sim 1 \mathrm{~mm}^{3}\right)$ [4], and is thought to be ignited by $\mathrm{Na}^{+} / \mathrm{K}^{+}$ATPase failure [5] or neuronal hyperexcitability [6]. SD then propagates radially, or along anatomical (i.e. sulci in gyrencephalic brains) or functional barriers (i.e. area undergoing seizure) at a rate of 2-6 $\mathrm{mm} / \mathrm{min}$ [7-9]. Over its course of propagation, SD is accompanied by a transient spreading depression of activity [10], tissue acidosis [11], cytotoxic edema [12], and a characteristic cerebral blood flow (CBF) response [13]. SD has been proposed to exacerbate ischemic or traumatic brain injury by deepening the metabolic crisis of tissue at risk $[14,15]$, and to correlate with unfavorable neurological outcome in these states [16-18]. In optimally perfused tissue, SD may not cause direct, gross injury [19], although SD has been implicated in the activation of trigeminal circuits and the evolution of migraine headache $[20,21]$, and the suspension of cardiorespiratory pacemaking in the brain stem in transgenic mouse models of SUDEP [3, 22].

The complexity and heterogeneity of conditions associated with SD in the living brain encouraged researchers to study SD in less complex model systems. In vitro live brain slice preparations have been suitable to recapitulate the neurophysiological hallmarks of SD accurately [23], and offer the opportunity to explore these changes free of cerebrovascular interferents [24]. The latter feature is especially pertinent when conducting optical imaging. Further, manipulations of the composition of the medium over the preparation allows the controlled simulation of global cerebral ischemia (e.g. oxygen glucose deprivation, OGD) [24], tissue acidosis (e.g. changing the bicarbonate or $\mathrm{CO}_{2}$ content of the medium) [25], or tissue swelling (e.g. hypo-osmotic medium, HM) [26]. Also, the application of pharmacological agents may yield highly reproducible, consistent data due to the uniform conditions, to aid the mechanistic understanding of SD evolution. For example, specific ion channels, exchangers or transporters mediating ion currents or amino acid translocations with SD may be studied effectively [27-31]. Finally, brain slice preparations are an ideal, basic experimental model to test the efficacy of SD inhibiting agents (e.g. NMDA or sigma-1 receptor blockers) with the goal to achieve neuroprotection [32, 33].

Even though a significant advantage of experimenting on live brain slices is the expected consistency of the results, there are methodological differences among laboratories, which complicate the integrative interpretation of data. The anatomical regions studied (cortex, hippocampus, striatum, subcortical gray matter or brain stem), the mode of SD initiation (e.g. high concentration $\mathrm{KCl}$ focal or global application, electric stimulation, transient hypoxia) or the type of the brain slice chamber used (interfaced or submerged preparations) may all modulate SD features in a manner specific for the given experimental approach [34].

Here we set out to provide a comparative evaluation of SD evolution in live brain slices, in response to selected SD triggers and in various media, under otherwise standardized experimental conditions. Local field potential (LFP) and intrinsic optical signal (IOS) imaging were applied for the assessment of the temporal and spatial characteristics of SD. This is the first study to explore the electrophysiological together with the intrinsic optical signal features of SDs under global oxygen-glucose deprivation or osmotic stress with respect to the physiological condition. The histological consequences of SD under these states are also evaluated. We pay particular attention here to spatial SD features, such as the volume of the area engaged in SD, which then corresponds to injury progression in the brain slice [31]. Rather than examining $\mathrm{SD}$ at the cellular level in a restricted tissue volume on the $\mu \mathrm{m}$-scale $[34,35]$, we provide large scale $(\mathrm{mm})$ whole slice measurements. The results are expected to aid the reliable reproduction of SD in live brain slice preparations, to serve the formulation of integrative conclusions.

\section{Results \\ Prolonged SDs occur upon acute osmotic stress and in response to oxygen glucose deprivation}

First, we compared the electrophysiological features of spontaneous SDs occurring in HM or during OGD to SDs evoked in normal artificial cerebrospinal fluid (aCSF). Prolonged SDs and incomplete direct current (DC) potential recovery were observed during OGD incubation and HM exposure compared to SDs evoked by $\mathrm{KCl}$ or electric stimulation (ES) in aCSF $(33.95 \pm 26.615$ vs. $54.75 \pm 26.72$ vs. $195.65 \pm 117.05$ vs. $112.14 \pm 88.39 \mathrm{~s}$, $\mathrm{KCl}$ vs. ES vs. HM vs. OGD) (Fig. 1b, d). The incomplete DC potential recovery from SD was also reflected by the reduced slope of repolarization in the OGD and HM groups compared to aCSF $(0.45 \pm 0.27$ vs. $0.50 \pm 0.24$ vs. $0.30 \pm 0.17$ vs. $0.12 \pm 0.10$ vs. $\mathrm{mV} / \mathrm{s} \mathrm{KCl}$ vs. ES vs. $\mathrm{HM}$ vs. OGD) (Fig. 1e). On the other hand, the greatest SD amplitudes were measured during HM exposure and ES $(-7.09 \pm 3.4$ vs. $-15.03 \pm 5.01$ vs. $-17.59 \pm 5.3$ vs. $-7.64 \pm 5.33 \mathrm{mV}, \mathrm{KCl}$ vs. ES vs. HM vs. OGD) (Fig. 1c).

\section{Severe osmotic stress and oxygen glucose deprivation enhance the area invaded by SD}

Next, we set out to explore whether longer SDs also invade larger tissue area, indicative of a larger bulk of tissue at risk of injury. To address this question, we performed IOS imaging of the brain slices. IOS imaging was also suitable to calculate the rate of SD propagation and 


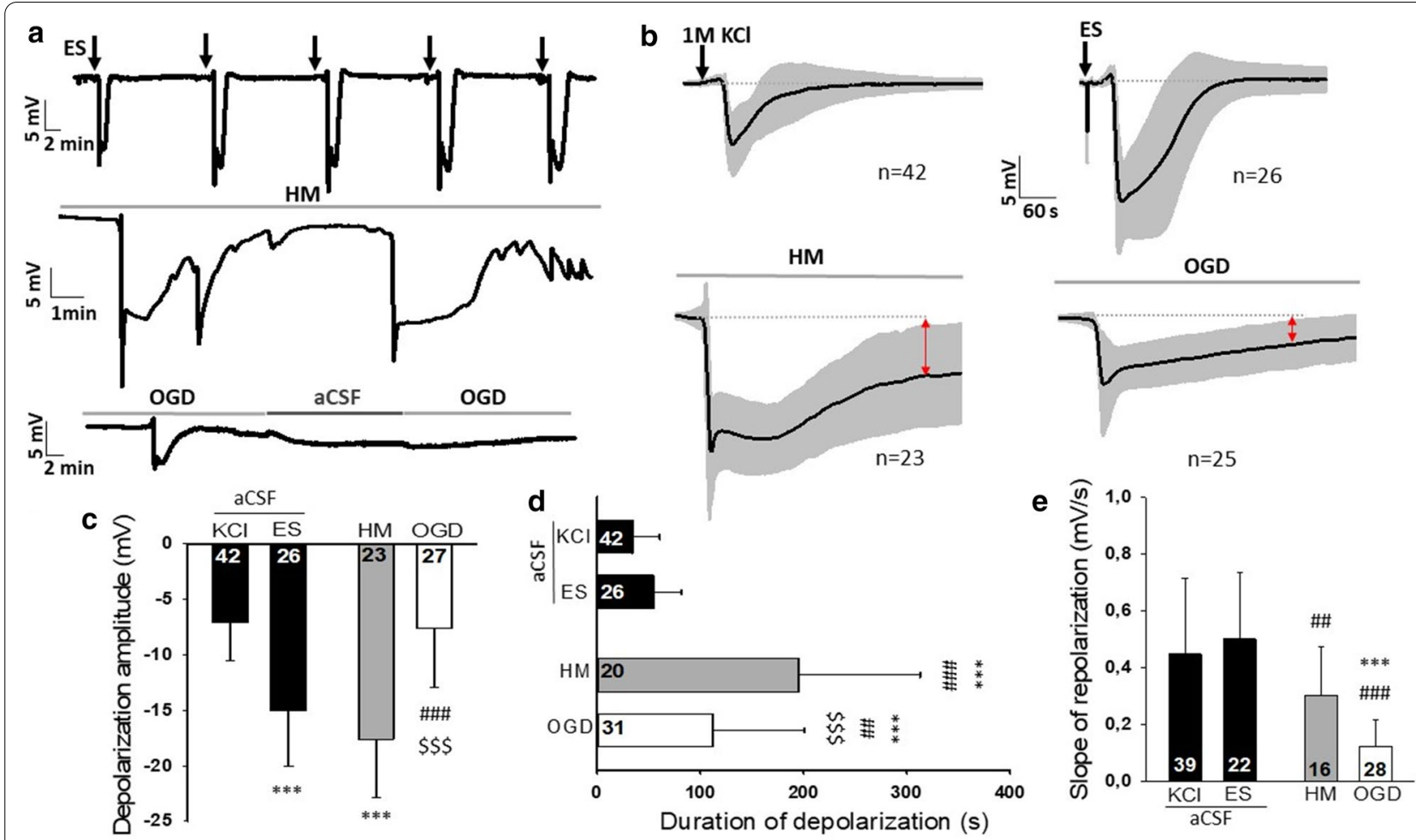

Fig. 1 Electrophysiological characterization of spreading depolarizations. a Representative DC potential recordings demonstrate the experimental protocols. Slices were exposed to aCSF (SD initiation here: ES), HM, or OGD. Black vertical arrows indicate ES in aCSF, gray horizontal timelines depict the exposure to the experimental condition (HM or OGD). b The SD-related negative DC shifts (mean \pm stdev) for each experimental group. Black vertical arrows indicate $\mathrm{KCl}$ application or ES in aCSF, gray horizontal timelines depict the exposure to the experimental condition (HM or OGD). Red arrows highlight incomplete repolarization relative to pre-SD baseline. $\mathbf{c}$ The maximum amplitude of SD. $\mathbf{d}$ The duration of SD. e The slope of repolarization. ES bipolar electric field stimulation, $\mathrm{KCl}$ microinjection of $1 \mathrm{M} \mathrm{KCl}$ solution, HM hypo-osmotic medium, OGD oxygen-glucose deprivation. Data are given as mean \pm stdev. Sample size (i.e. number of SD events analyzed) is indicated in each bar. One-way analysis of variance (ANOVA) followed by a Holm-Sidak post hoc test was used for statistical analysis. The level of significance is given as $p<0.001^{* * *} \mathrm{vs}$. KCl; $p<0.01^{\# \#}$

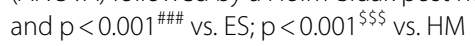

SD latency (i.e. the time between the onset of HM or OGD incubation and the appearance of the SD focus).

The SD latency with respect to the onset of treatment was much longer in slices exposed to HM than to OGD $(549.1 \pm 164.59$ vs. $165.75 \pm 52.3$ s, HM vs. OGD), while SD occurred within seconds after $\mathrm{KCl}$ or ES elicitation in aCSF (Fig. 2e). SDs during OGD or HM exposure propagated at a higher rate than SDs induced by $\mathrm{KCl}$ or ES in aCSF $(1.7 \pm 0.59$ vs. $1.94 \pm 0.73$ vs. $4.24 \pm 1.32$ vs. $2.15 \pm 0.83 \mathrm{~mm} / \mathrm{min}, \mathrm{KCl}$ vs. ES vs. HM vs. OGD) (Fig. 2c).

The tissue area representing the SD focus was enlarged during OGD or HM incubation compared to ES or $\mathrm{KCl}$-evoked SDs in aCSF $(1.37 \pm 0.5$ vs. $1.93 \pm 0.74$ vs. $2.64 \pm 1.27$ vs. $2.23 \pm 0.82 \%$ of the total cortical area, $\mathrm{KCl}$ vs. ES vs. HM vs. OGD) (Fig. 2a). SDs also invaded a significantly larger cortical area in the OGD and in the HM conditions compared to aCSF (36.72 \pm 9.39 vs. $40.4 \pm 20.66$ vs. $71.98 \pm 13.43$ vs. $85.32 \pm 5.32 \%$ of total cortical area, $\mathrm{KCl}$ vs. ES vs. HM vs. OGD) (Fig. 2a, b).
SDs initiated with a focal stimulus ( $\mathrm{KCl}$ and $\mathrm{ES}$ ) obviously evolved at the site of trigger delivery. During HM treatment or OGD, depolarization foci occurred in the parietal and temporal cortex (Fig. 2d).

Furthermore, SDs also occurred in the striatum during OGD in all slices examined $(n=9)$ (Fig. 2a, d). In HM, SD emerged in the striatum in 4 of 9 slices, and cortical SDs invaded the striatum from the ventral tip of the cortex in an additional 3 slices (Fig. 2d). The SDs with a striatal focus occurred invariably after the cortical SDs in these experiments, with a latency of 1-2 min with respect to the generation of the cortical SDs $(34.85 \pm 7.21$ and $137.89 \pm 59.39$ s HM and OGD).

\section{Oxygen glucose deprivation and osmotic stress restrain cell survival after SD}

Finally, we moved on to analyze the histological outcome after the passage of SDs. 2,3,5-triphenyltetrazolium chloride (TTC) staining commonly used for the detection of lesion development [36-38] was applied to quantify the 


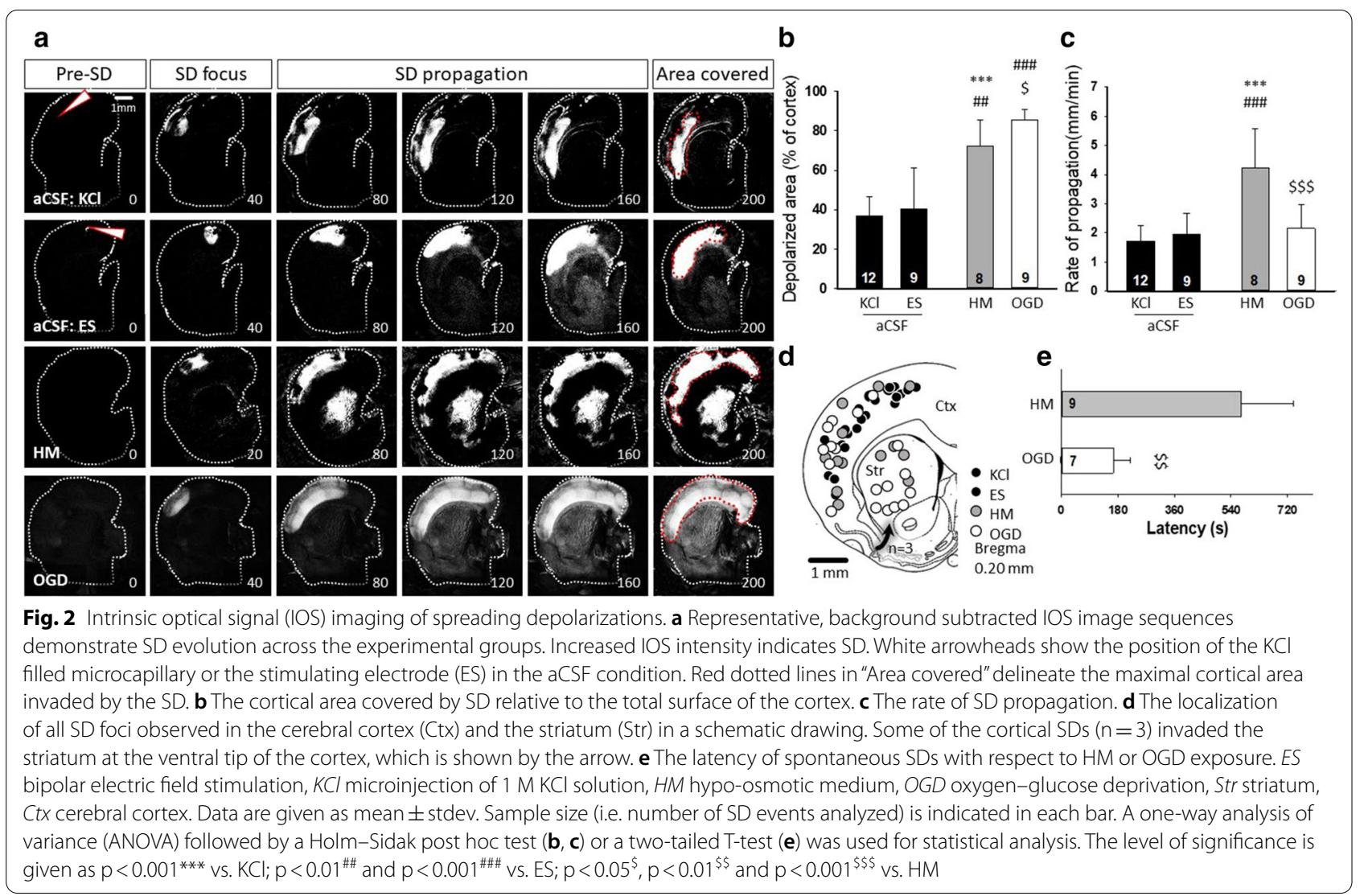

SD-related tissue injury. We accepted higher numbers of TTC-positive cellular compartments (i.e. particles) to indicate better tissue viability [31]. The SD-related TTC-positive particle loss was obvious after the single SD in HM or OGD $(26.45 \pm 10.64$ and $11.44 \pm 6.42$ vs. $54.33 \pm 21.21$ per $1000 \mu \mathrm{m}^{2}, \mathrm{HM}$ and OGD vs. $\left.\mathrm{KCl}\right)$. Also, the repeated elicitation of SD with ES in aCSF caused a significant reduction in particle number $(30.28 \pm 9.84$ vs. $54.33 \pm 21.21$ per $1000 \mu \mathrm{m}^{2}$, ES vs. KCl) (Fig. 3a, b). Corresponding neuronal injury was observed in hematoxylin-eosin-stained sections: condensed, fragmented nuclei and vacuolization indicated pronounced neuronal damage after OGD and HM treatment (Fig. 3a). This was also seen to a lesser degree after recurrent SDs triggered with ES. In contrast, neurons were preserved and had a large, round nucleus with a prominent nucleolus after repeated, $\mathrm{KCl}$ evoked SDs (Fig. 3a). Finally, a linear negative correlation was found between the number of TTC-positive particles and the size of the cortical area engaged in an SD (Fig. 3c).

\section{Discussion}

In the present study, our goal was the comparative evaluation of SD evolution in rat coronal brain slices exposed to osmotic or metabolic stress. SDs elicited with the focal application of $1 \mathrm{M} \mathrm{KCl}$ or bipolar electric stimulation in slices bathed in aCSF were used as reference. The application of OGD to study SD was justified by its widespread use to mimic severe global ischemia (e.g. cardiac arrest) or conditions prevalent in the ischemic core in focal cerebral ischemia [24, 39]. Of note, the less commonly implemented, partial metabolic challenge of submerged slices may be relevant to recapitulate ischemic penumbra conditions, although this model was reported not to give rise to spontaneous SDs [33]. Further, hypoxia at normal glucose levels has long been known to trigger SD, but this condition should not be confused with ischemia (i.e. ischemia is the restricted availability of oxygen, as well as glucose) [40]. To study the impact of osmotic stress on SD, we incubated the brain slices in HM, following a previously established approach to model cerebral edema [26, 31]. We experimented with HM solutions of decreasing $\mathrm{Na}^{+}$content in our pilot experiments (120100-80-60-40 mM, $\mathrm{HM}_{120}-\mathrm{HM}_{40}$, respectively), and selected here $\mathrm{HM}_{60}$ for systematic work, because incubation of the slices in $\mathrm{HM}_{60}$ reliably produced SD within 10 min after $\mathrm{HM}$ application. Higher $\mathrm{Na}^{+}$concentration $\mathrm{HM}$ solutions in our pilot experiments (e.g. $\mathrm{HM}_{100}$ ) also caused SD occurrence, but at more variable and typically longer latency. The used $\mathrm{Na}^{+}$concentration may occur 


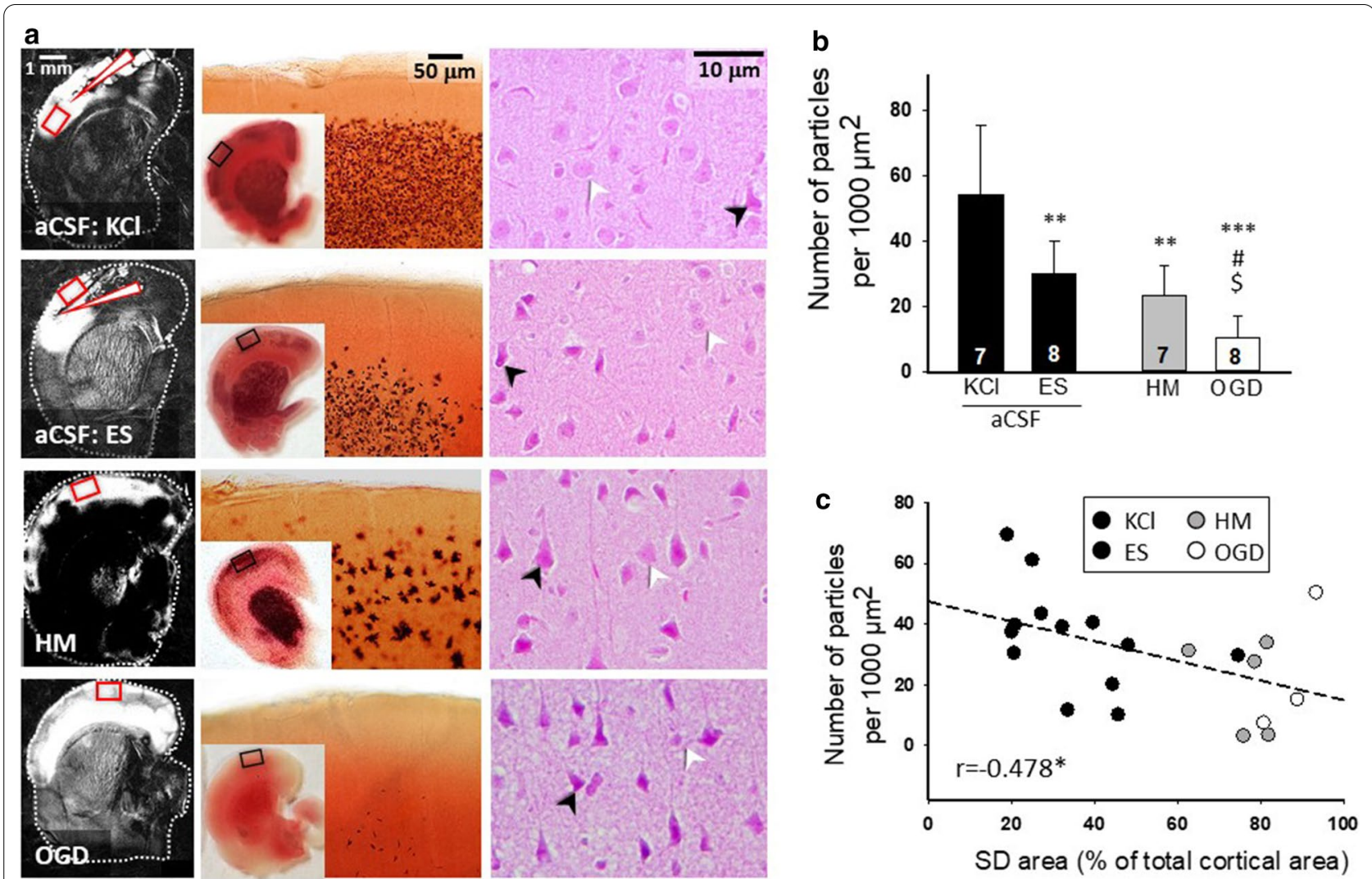

Fig. 3 Histological analysis of SD-related tissue injury. a Representative background subtracted IOS images to the left show the surface of the cortex covered by the propagating SD (bright region). White arrowheads are pointing at the position of the site of SD elicitation with $\mathrm{KCl}$ or ES. In the middle, representative light microscopic images of brain slices stained with TTC after the passage of SDs (4-4 SDs were elicited in each slice with $\mathrm{KCl}$ or ES; a single SD occurred in response to HM or OGD) show macroscopic injury. Representative photomicrographs of hematoxylineosin-stained sections to the right demonstrate neuronal injury. Black arrowheads indicate injured neurons; white arrowheads show viable cells with intact nuclei in the somatosensory cortex. $\mathbf{b}$ The number of TTC-stained cellular compartments (i.e. particles). c Correlation between the number of particles and the cortical area bearing an SD as in Fig. 2b. Data are given as mean \pm stdev. Sample size (i.e. number of slices analyzed) is indicated in each bar. ES bipolar electric field stimulation, $\mathrm{KCl}$ microinjection of $1 \mathrm{M} \mathrm{KCl}$ solution, HM hypo-osmotic medium, OGD oxygen-glucose deprivation. Statistical analysis relied on one-way analysis of variance (ANOVA) followed by a Holm-Sidak post hoc test (b), or one-tailed Pearson

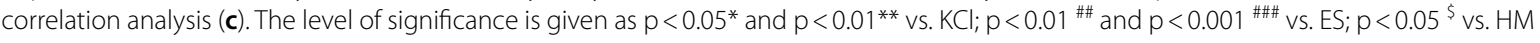

spatio-temporally locked with SD in the in vivo brain, as the extracellular concentration of $\mathrm{Na}^{+}$has been known to decrease from $140-150 \mathrm{mM}$ down to $50-70 \mathrm{mM}$ with SD [41]. The SD-linked osmotic stress, however, must be less pronounced, due to the compensatory accumulation of osmolites other than $\mathrm{Na}^{+}$in the interstitium (e.g. $\mathrm{K}^{+}$ and glutamate) $[41,42]$. Finally, as control for both OGD and $\mathrm{HM}$, we elicited SD in aCSF with highly focused $\mathrm{KCl}$ pressure injection (picoliter volume), expected to be near the minimum conditions of SD elicitation [34], and the least invasive. This condition is thought to be relevant to model SD as it occurs in migraine with aura [43]. In other aCSF-incubated slices, electrical stimulation was applied for SD elicitation, due to its frequent application in a number of labs with the purpose to determine the threshold of SD elicitation $[44,45]$. The otherwise standardized conditions (i.e. the level of the coronal brain slices, the region studied, the composition and temperature of the media, the type of the brain slice chamber and the standard approach of data acquisition) allowed the identification of SD features that occur typically in response to these well-defined and reliably reproducible osmotic or ischemic challenges.

Both OGD and HM gave rise to a spontaneous, longlasting SD, at a short latency as previously shown (2.7$9.2 \mathrm{~min})[35,46]$. These spontaneous events propagated over the entire cortical surface in the preparation. The brain slice was irresponsive to further stimulation after SD in OGD (Fig. 1), and displayed acute, extensive histological damage in the path of SD (Fig. 3), therefore SD has been accepted as a terminal event in the cortex in OGD. These results agree with earlier findings [46, 47]. In contrast, the tissue repolarized from the SD in HM - albeit with a long delay (Fig. 1)-confirming previous 
observations [35, 48]. The partial electrophysiological regeneration was supported by the propensity of the tissue to bear subsequent SDs either spontaneously occurring in HM (Fig. 1) or triggered with transient anoxia in HM [31]. In line with the functional data, the first SD in HM caused cellular injury in the cortex engaged in $\mathrm{SD}$, but the damage was less severe than that seen after SD in OGD (Fig. 3). SD evoked by OGD sustained for 15-20 min here was associated with extensive injury in all layers of the cortex as evidenced by TTC staining (Fig. 3a), and in line with previous reports [46, 47]. Yet, some cortical neurons (e.g. in layer 4) may survive shorter OGD (about $10 \mathrm{~min}$ ) followed by reperfusion with normal aCSF, and not reach a commitment point to neuronal death, as shown by patch clamp recordings [49]. Also, subcortical structures, such as neuron populations in the hypothalamus and the brain stem may be more resistant to OGD than the cortex $[47,50]$.

In the control conditions here ( $\mathrm{KCl}$ or $\mathrm{ES})$, a train of 4-5 short-lasting SDs could be elicited at an interval of 10-12 min in agreement with previous reports [32], which demonstrates the sustained viability of the tissue after the passage of SD under physiological conditions. Of the two control conditions here, focal low volume $\mathrm{KCl}$ application with pressure injection caused no detectable histological degeneration in the brain slice. However, EStriggered SDs were accompanied with obvious damage to the tissue. Although not documented systematically, most SD researchers agree that electric stimulation with a charge sufficient to trigger SD leaves focal necrotic tissue damage behind at the electrode contact point [51]. This is also substantiated by the need to reposition the stimulating electrode between SDs evoked to estimate the electric SD threshold reliably [45]. Collectively, our results here present three stages of the SD continuum [51]: (i) apparently harmless SD in intact tissue followed by sufficient recovery, (ii) injurious SD with poor recovery emerging under osmotic stress, and (iii) terminal and detrimental $\mathrm{SD}$ in oxygen and glucose deprived tissue.

The distinct features of the negative DC shift and IOS signature of SD may disclose some mechanistic elements of SD evolution typical for the homeostatic conditions. For example, the slower rate of repolarization in HM and OGD as seen here (Fig. 2) is thought to correspond to the dysfunctional re-uptake of $\mathrm{K}^{+}$or glutamate. The shortage of ATP in OGD causes the failure of the neuronal and astrocytic $\mathrm{Na}^{+} / \mathrm{K}^{+}$ATP-ase, which hampers the uptake of $\mathrm{K}^{+}$accumulated with the SD wave front in the extracellular space $[5,14]$. Although osmotic stress could also attenuate the enzymatic activity of the $\mathrm{Na}^{+} / \mathrm{K}^{+}$ATP-ase [52], HM exposure has been shown to induce prominent astrocyte swelling [31, 53] and to disrupt the astrocyte buffer capacity, reflected by a progressive accumulation of glutamate, for example [31]. Astrocytic $\mathrm{K}^{+}$and glutamate re-uptake has been implicated in the repolarization after SD [54]. Taken together, we propose that the plausible cause of slower repolarization and prolonged SD duration in $\mathrm{HM}$ was the injured glial $\mathrm{K}^{+}$clearance and glutamate transport. The failure of the $\mathrm{Na}^{+} / \mathrm{K}^{+}$ATP-ase and the exhaustion of the astroglial buffer capacity are also suspected to contribute to the remarkably greater area engaged in SD propagation in HM and OGD compared to aCSF (Fig. 2).

SD in HM stood out from the other experimental conditions with its high rate of propagation and DC shift amplitude (Fig. 2). A recent study of ours has conducted extensive pharmacological manipulations and electrophysiological measurements to understand the mechanistic background of SD evolution in HM [31]. We have demonstrated augmented glutamate accumulation, a profound increment of evoked field potential amplitude and decreased electric threshold of SD elicitation in HM [31], which appear to be consistent with the enhanced neuronal excitability and intensified synaptic activity under osmotic stress $[35,55]$. In addition, the shrinkage of the extracellular space in HM [26] may also account for the accelerated propagation rate of SD.

\section{Conclusions and perspectives}

In summary, we show here that under otherwise standardized experimental conditions, SD is most pronounced in HM, in which condition the metabolic substrate supply is unlimited, but the tissue is hyper excitable due to cellular swelling and impaired glutamate clearance. In contrast with OGD, SD propagating under hypo-osmotic condition is not terminal, yet it is associated with irreversible tissue injury. This in vitro study offers a new perspective by the direct comparison of these two states, which must co-exist and intertwine in acute brain injury.

Finally, in vitro models, as presented here, offer an opportunity to screen the neuroprotective potential of therapeutically promising drug candidates, before testing them in in vivo systems. The pharmacological modulation of neurophysiological events that take place in the first minutes after acute brain injury may not seem clinically relevant. Yet, it must be realized that with the maturation of the injury, SDs become recurrent and at least some of them originating at the peri-infarct zone share basic features with the initial SD [1]. Also, to substantiate the value of OGD or HM brain slice studies: the pharmacological delay of SD onset in the OGD model proved to be a first step to later identify pertinent drug effect achieved by targeting sigmal receptors [56, 57]. Therefore, experimentation in these simple model systems is expected to impart valuable understanding of SD 
pathophysiology, effectively complement in vivo work, and identify potential sites for intervention.

\section{Methods}

The experimental procedures were approved by the National Food Chain Safety and Animal Health Directorate of Csongrád-Csanád County, Hungary. The procedures were performed according to the guidelines of the Scientific Committee of Animal Experimentation of the Hungarian Academy of Sciences [updated Law and Regulations on Animal Protection: 40/2013. (II. 14.) Gov. of Hungary], following the EU Directive 2010/63/EU on the protection of animals used for scientific purposes, and reported in compliance with the ARRIVE guidelines.

Young adult, male Sprague-Dawley and Wistar rats (body weight: $250 \mathrm{~g} ; \mathrm{n}=51$ ) were used in this study. Animals were acquired from the Central Animal House of Biological Research Center, Szeged (Charles River Laboratories breed) and the animal house of the Department of Pharmacodynamics and Biopharmacy, University of Szeged (Charles River Laboratories breed). Standard rodent chow and tap water were supplied ad libitum. The animals were housed under constant temperature, humidity, and lighting conditions $\left(23{ }^{\circ} \mathrm{C}, 12: 12 \mathrm{~h}\right.$ light/ dark cycle, lights on at 7 a.m.).

\section{Live brain slice preparations}

The procedures of live brain slice preparation were identical to those reported earlier [30]. The brains of the rats were removed under deep anesthesia (4-5\% isoflurane in $\left.\mathrm{N}_{2} \mathrm{O}: \mathrm{O}_{2} ; 2: 1\right)$. Coronal brain slices $(350 \mu \mathrm{m})$ anterior to bregma were cut with a vibrating blade microtome (Leica VT1000S, Leica, Germany), and collected in icecold aCSF (composition of aCSF in mM concentrations: $130 \mathrm{NaCl}, 3.5 \mathrm{KCl}, 1 \mathrm{NaH}_{2} \mathrm{PO}_{4}, 24 \mathrm{NaHCO}_{3}, 1 \mathrm{CaCl}_{2}, 3$ $\mathrm{MgSO}_{4}$ and 10 Dglucose). Four to six slices were transferred to an incubation chamber filled with carbogenated aCSF (difference in composition in $\mathrm{mM}$ concentrations: $3 \mathrm{CaCl}_{2}$ and $1.5 \mathrm{MgSO}_{4}$ ) and kept at room temperature $\left(\sim 20-22{ }^{\circ} \mathrm{C}\right)$. Individual slices were placed in an interface type recording tissue chamber (Brain Slice Chamber BSC1, Scientific Systems Design Inc., Ontario, Canada) and continuously perfused with carbogenated aCSF at a rate of $2.5 \mathrm{ml} / \mathrm{min}$. Chamber temperature was kept at $32{ }^{\circ} \mathrm{C}$ using a dedicated proportional temperature controller unit (PTC03, Scientific Systems Design Inc., Ontario, Canada) [30].

\section{Local field potential recordings}

LFP filtered in DC mode $(<1 \mathrm{~Hz})$ was acquired via a glass capillary microelectrode (1-3 M $\Omega$ ) filled with $150 \mathrm{mM}$ $\mathrm{NaCl}$ and $1 \mathrm{mM}$ HEPES. The microelectrode was inserted into the 3rd cortical layer, and an $\mathrm{Ag} / \mathrm{AgCl}$ reference electrode was positioned in the recording chamber. The glass capillary microelectrode was connected to a custom-made dual-channel electrometer (including AD549LH, Analog Devices, Norwood, MA, USA), and the signal was fed to dedicated differential amplifiers and associated filter modules (NL106 and NL125, NeuroLog System, Digitimer Ltd., United Kingdom). The recorded analogue signal was converted to digital signal and displayed live using an Acqknowledge environment (MP 150, Biopac Systems, Inc) at a sampling frequency of $1 \mathrm{kHz}$ [30]. DC potential traces confirmed the occurrence of SD events. Further, the DC potential recordings were used off-line to determine the amplitude, duration at half amplitude and the slope of depolarization and repolarization of SDs.

\section{Intrinsic optical signal imaging}

For IOS imaging, slices were illuminated by a halogen lamp (Volpi AG, Intralux 5100, Schlieren, Switzerland). Image sequences were captured at $1 \mathrm{~Hz}$ with a monochrome CCD camera (spatial resolution: $1024 \times 1024$ pixel, Pantera 1M30, DALSA, Gröbenzell, Germany) attached to a stereomicroscope (MZ12.5, Leica Microsystems, Wetzlar, Germany), yielding 6-10× magnification. Image sequences were analyzed off-line; the area of the $\mathrm{SD}$ focus, the total area covered by the propagating SD, and the propagation velocity of SDs were analyzed after contrast enhancement in Fiji.

\section{Methods of SD elicitation $\mathrm{KCl}$ injection}

SDs were evoked by pressure injection of $1 \mathrm{M} \mathrm{KCl} \mathrm{(40}$ ms, 30 psi, approximately 150 picoliter) via a glass micropipette, using a picospritzer (40 ms, 30 psi, Picospritzer III, Parker Hannifin, Hollis, USA). The micropipette was lowered into the 3rd cortical layer at a distance of $500 \mu \mathrm{m}$ from the recording electrode. Four-to-five successive SDs were initiated in each slice at 10-12 min intervals $(\mathrm{n}=38)$.

\section{Electric stimulation}

In order to elicit SD with ES, a concentric bipolar needle electrode (WPI, Sarasota, FL, USA) was positioned onto brain slices, at a distance of approximately $800-1000 \mu \mathrm{m}$ from the DC potential recording electrode. The elicitation of SD followed previously established principles [45]. The stimulating electrode was connected to an opto-coupled stimulus isolator with constant current output (NL 800, Digitimer Ltd., United Kingdom), a pulse generator (NL301), a width-delay panel (NL405), and a pulse buffer (NL510), which enabled the adjustment of the duration of the stimuli at will. Stimulation was implemented with single, cathodal constant current pulses. The charge 
delivered was increased stepwise until SD was initiated, quantified as $\mathrm{Q}[\mu \mathrm{C}]=\mathrm{I}[\mathrm{mA}] \times \mathrm{t}[\mathrm{ms}]$, and ranged between 50-100 $\mu \mathrm{C}$. Four-to-five consecutive SDs were initiated in each slice at 10-12 min intervals to allow full recovery between events $(n=51)$.

\section{Hypo-osmotic challenge}

Slices $(\mathrm{n}=31)$ were exposed to HM, which were prepared by reducing the $\mathrm{NaCl}$ concentration of aCSF from the regular $130 \mathrm{mM}$ to $60 \mathrm{mM}$, while other components and the $\mathrm{pH}$ of the medium were unaltered. Electrophysiological recording or IOS imaging was initiated during normal aCSF incubation, 10-15 min before switching to HM superfusion, and pursued 15-20 min into HM incubation. SD occurred spontaneously in response to osmotic stress.

\section{Oxygen-glucose deprivation}

OGD was induced by super-fusion of glucose-free aCSF (glucose was substituted with sucrose at equimolar concentration) on slices $(\mathrm{n}=40)$, and the solution was gassed with $95 \% \mathrm{~N}_{2}+5 \% \mathrm{CO}_{2}$. Electrophysiological recording or IOS imaging was initiated during normal aCSF incubation, 10-15 min before OGD onset, and pursued for 15-20 min after OGD onset. OGD invariably gave rise to an SD event. In selected slices, aCSF incubation was resumed after OGD for $5 \mathrm{~min}$, and OGD was imposed again for $30 \mathrm{~min}$, to test whether a subsequent SD event could generate (Fig. 1).

\section{Histology \\ TTC staining}

The size of the ischemic lesion was determined by TTC staining. The brain slices $(n=30)$ were incubated in a $2 \%$ solution of TTC in $0.1 \mathrm{M}$ phosphate buffered saline (PBS) for $20 \mathrm{~min}$ at room temperature. The sections were subsequently immersed and stored in $4 \%$ paraformaldehyde for $24 \mathrm{~h}$. The stained sections were then mounted on microscope slides and coverslipped with glycerol. Images were taken of the cortex with Nikon-DS Fi3 camera attached to a Leica DM 2000 Led light microscope (Leica Microsystems $\mathrm{GmbH}$, Germany) at 10× magnification.

\section{Hematoxylin-eosin staining}

For proper visualization of cell damage, representative TTC stained slices were rinsed with PBS and cryoprotected in $30 \%$ sucrose in PBS. Coronal, $10-\mu \mathrm{m}$-thick frozen sections were cut with a freezing microtome (Leica CM 1860 UV, Leica, Germany). The sections were stained with hematoxylin (Sigma-Aldrich, USA) for $25 \mathrm{~s}$, rinsed with distilled water, then stained with eosin (Sigma-Aldrich, USA) for $15 \mathrm{~s}$, rinsed with distilled water, dehydrated, and coverslipped with Eukit ${ }^{\circledR}$ (Merck, USA).
The sections were examined with optical microscopy; photomicrographs at $40 \times$ magnification were taken with a Nikon-DS Fi3 camera attached to a Leica DM 2000 Led light microscope (Leica Microsystems GmbH, Germany).

\section{Data analysis}

LFP traces were stored using a personal computer equipped with a dedicated software (AcqKnowledge 4.2 for MP 150, Biopac Systems, Inc., USA). Data analysis was conducted offline and was assisted by the inbuilt tools of AcqKnowledge 4.2 software.

Spatial features of SDs were measured in image sequences after background subtraction and manual thresholding in Fiji. The area of the SD focus and the total area covered by the propagating SD were expressed relative to the full surface area of the cortex in the brain slice. The calculation of the propagation velocity was aided by the IOS signal and was more accurate due to the perceptible direction of SD propagation. SD events with amplitude $<3 \mathrm{mV}$ or those where the post-SD DC signal remained level were excluded from data analysis.

To characterize SD events, the amplitude, the slope of depolarization and repolarization, and the duration of SD were quantitated in the LFP traces filtered in DC mode. These variables are conventionally taken as a measure of the propensity of the tissue to bear SD (e.g. slower depolarization or smaller amplitude disclose lower SD susceptibility) $[11,58,59]$, and to predict the injurious potential of SD (the duration of SD or the cumulative duration of recurrent SDs correlate with the degree of ischemic injury) $[16,60]$. The duration of SD may also disclose the severity of the ongoing injurious condition. As such, SD becomes remarkably prolonged or terminal when the metabolic crisis in the tissue is severe [14].

After binary conversion, TTC stained images were manually thresholded, particles were measured with the inbuilt "analyze particles" function of FIJI and expressed as \% area of baseline.

Quantitative data are given as mean \pm standard deviation (stdev). Statistical analysis was conducted with the software SigmaPlot 13.0 (Systat Software, Inc. San Jose, USA). Data sets were evaluated by two-tailed T-test or ANOVA followed by a Holm-Sidak post hoc test, or onetailed Pearson correlation. Levels of significance were set at $\mathrm{p}<0.05$.

\section{Abbreviations}

aCSF: Artificial cerebrospinal fluid; ANOVA: One-way analysis of variance; CBF: Cerebral blood flow; DC: Direct current; ES: Electric stimulation; HM: Hypoosmotic medium; IOS: Intrinsic optical signal; LFP: Local field potential; OGD: Oxygen glucose deprivation; PBS: Phosphate buffered saline; SD: Spreading depolarization; SUDEP: Sudden unexpected death in epilepsy; TTC: 2,3,5-Triphenyltetrazolium chloride. 


\author{
Acknowledgements \\ The authors are grateful to Szilvia V. Kecskés for histological preparations.
}

\section{Authors' contributions}

RF: acquisition of data, analysis and interpretation of data, drafting the article: FB: revising the article critically for important intellectual content; ÁM: design of the study, analysis and interpretation of data, revising the article critically for important intellectual content; EF: design of the study, analysis and interpretation of data, drafting the article. All authors read and approved the final manuscript.

\section{Funding}

This work was supported by grants from the National Research, Development and Innovation Office of Hungary (K134377; K120358, and PD128821); the Economic Development and Innovation Operational Programme in Hungary co-financed by the European Union and the European Regional Development Fund (No. GINOP-2.3.2-15-2016-00006), and the EU-funded Hungarian grant No. EFOP-3.6.1-16-2016-00008.

\section{Availability of data and materials}

The datasets used and/or analyzed during the current study are available from the corresponding authors on reasonable request.

\section{Declarations}

\section{Ethics approval and consent to participate}

The experimental procedures were approved by the National Food Chain Safety and Animal Health Directorate of Csongrád-Csanád County, Hungary. The procedures were performed according to the guidelines of the Scientific Committee of Animal Experimentation of the Hungarian Academy of Sciences (updated Law and Regulations on Animal Protection: 40/2013. (II. 14.) Gov. of Hungary), following the EU Directive 2010/63/EU on the protection of animals used for scientific purposes, and reported in compliance with the ARRIVE guidelines.

\section{Consent for publication}

All authors have approved the final version of the manuscript.

\section{Competing interests}

The authors declare no competing interests.

Received: 13 February 2021 Accepted: 20 April 2021

Published online: 03 May 2021

\section{References}

1. Hartings JA, Shuttleworth CW, Kirov SA, Ayata C, Hinzman JM, Foreman $B$, et al. The continuum of spreading depolarizations in acute cortical lesion development: Examining Leao's legacy. J Cereb Blood Flow Metab. 2017;37(5):1571-94

2. Brennan KC, Pietrobon D. A systems neuroscience approach to migraine. Neuron. 2018;97(5):1004-21.

3. Aiba I, Noebels JL. Spreading depolarization in the brainstem mediates sudden cardiorespiratory arrest in mouse SUDEP models. Sci Transl Med. 2015;7(282):282ra46.

4. Matsuura T, Bures J. The minimum volume of depolarized neural tissue required for triggering cortical spreading depression in rat. Exp Brain Res. 1971;12(3):238-49.

5. Reiffurth C, Alam M, Zahedi-Khorasani M, Major S, Dreier JP. $\mathrm{Na}(+) / \mathrm{K}(+)$-ATPase alpha isoform deficiency results in distinct spreading depolarization phenotypes. J Cereb Blood Flow Metab. 2020;40(3):622-38.

6. Vinogradova LV. Initiation of spreading depression by synaptic and network hyperactivity: Insights into trigger mechanisms of migraine aura. Cephalalgia Int J Headache. 2018;38(6):1177-87.

7. Leao A. Spreading depression of activity in the cerebral cortex. J Neurophysiol. 1944;7: 359-390.
8. Koroleva VI, Bures J. Circulation of cortical spreading depression around electrically stimulated areas and epileptic foci in the neocortex of rats. Brain Res. 1979:173(2):209-15.

9. Santos E, Scholl M, Sanchez-Porras R, Dahlem MA, Silos H, Unterberg A, et al. Radial, spiral and reverberating waves of spreading depolarization occur in the gyrencephalic brain. Neurolmage. 2014;99:244-55.

10. Dreier JP, Fabricius M, Ayata C, Sakowitz OW, Shuttleworth CW, Dohmen $C$, et al. Recording, analysis, and interpretation of spreading depolarizations in neurointensive care: Review and recommendations of the COSBID research group. J Cereb Blood Flow Metab. 2017;37(5):1595-625.

11. Tóth MO, Menyhart A, Frank R, Hantosi D, Farkas E, Bari F. Tissue acidosis associated with ischemic stroke to guide neuroprotective drug delivery. Biology. 2020;9(12):460.

12. Dreier JP, Lemale CL, Kola V, Friedman A, Schoknecht K. Spreading depolarization is not an epiphenomenon but the principal mechanism of the cytotoxic edema in various gray matter structures of the brain during stroke. Neuropharmacology. 2018;134(Pt B):189-207.

13. Ayata C, Lauritzen M. Spreading depression, spreading depolarizations, and the cerebral vasculature. Physiol Rev. 2015;95(3):953-93.

14. Dreier JP. The role of spreading depression, spreading depolarization and spreading ischemia in neurological disease. Nat Med. 2011;17(4):439-47.

15. Hoffmann U, Ayata C. Neurovascular coupling during spreading depolarizations. Acta Neurochirurgica Suppl. 2013;115:161-5.

16. Dreier JP, Woitzik J, Fabricius M, Bhatia R, Major S, Drenckhahn C, et al. Delayed ischaemic neurological deficits after subarachnoid haemorrhage are associated with clusters of spreading depolarizations. Brain J Neurol. 2006;129(Pt 12):3224-37.

17. Hartings JA, Bullock MR, Okonkwo DO, Murray LS, Murray GD, Fabricius M, et al. Spreading depolarisations and outcome after traumatic brain injury: a prospective observational study. Lancet Neurol. 2011;10(12):1058-64.

18. Hartings JA, Andaluz N, Bullock MR, Hinzman JM, Mathern B, Pahl C, et al. Prognostic value of spreading depolarizations in patients with severe traumatic brain injury. JAMA Neurol. 2020;77(4):489-99.

19. Nedergaard M, Hansen AJ. Spreading depression is not associated with neuronal injury in the normal brain. Brain Res. 1988;24(1-2):395-8.

20. Karatas H, Erdener SE, Gursoy-Ozdemir Y, Lule S, Eren-Kocak E, Sen ZD, et al. Spreading depression triggers headache by activating neuronal Panx1 channels. Science. 2013:339(6123):1092-5.

21. Harriott AM, Chung DY, Uner A, Bozdayi RO, Morais A, Takizawa T, et al. Optogenetic spreading depression elicits trigeminal pain and anxiety behavior. Ann Neurol. 2021;89(1):99-110.

22. Loonen ICM, Jansen NA, Cain SM, Schenke M, Voskuyl RA, Yung AC, et al. Brainstem spreading depolarization and cortical dynamics during fatal seizures in Cacna1a S218L mice. Brain J Neurol. 2019;142(2):412-25.

23. Somjen GG. Mechanisms of spreading depression and hypoxic spreading depression-like depolarization. Physiol Rev. 2001;81(3):1065-96.

24. Joshi I, Andrew RD. Imaging anoxic depolarization during ischemialike conditions in the mouse hemi-brain slice. J Neurophysiol. 2001;85(1):414-24

25. Tong CK, Chesler M. Modulation of spreading depression by changes in extracellular pH. J Neurophysiol. 2000;84(5):2449-57.

26. Chebabo SR, Hester MA, Jing J, Aitken PG, Somjen GG. Interstitial space, electrical resistance and ion concentrations during hypotonia of rat hippocampal slices. J Physiol. 1995;487(Pt 3):685-97.

27. Czeh G, Aitken PG, Somjen GG. Membrane currents in CA1 pyramidal cells during spreading depression (SD) and SD-like hypoxic depolarization. Brain Res. 1993;632(1-2):195-208.

28. Dietz RM, Kiedrowski L, Shuttleworth CW. Contribution of $\mathrm{Na}(+) / \mathrm{Ca}(2+)$ exchange to excessive $\mathrm{Ca}(2+)$ loading in dendrites and somata of CA1 neurons in acute slice. Hippocampus. 2007;17(11):1049-59.

29. Wu DC, Chen RY, Cheng TC, Chiang YC, Shen ML, Hsu LL, et al. Spreading depression promotes astrocytic calcium oscillations and enhances gliotransmission to hippocampal neurons. Cereb Cortex. 2018;28(9):3204-16.

30. Menyhart A, Farkas AE, Varga DP, Frank R, Toth $R$, Balint AR, et al. Largeconductance $\mathrm{Ca}(2+)$-activated potassium channels are potently involved in the inverse neurovascular response to spreading depolarization. Neurobiol Dis. 2018;119:41-52.

31. Menyhárt Á, Frank R, Farkas AE, Süle Z, Varga VÉ, Nyúl-Tóth Á, Meiller A, Ivánkovits-Kiss O, Lemale CL, Szabó I , Tóth R, Zölei-Szénási D, Woitzik J, Marinesco S, Krizbai IA, Bari F, Dreier JP, Farkas E. Malignant astrocyte 
swelling and impaired glutamate clearance drive the expansion of injurious spreading depolarization foci. bioRxiv 2020.10.02.324103. https://doi. org/10.1101/2020.10.02.324103

32. Anderson TR, Andrew RD. Spreading depression: imaging and blockade in the rat neocortical brain slice. J Neurophysiol. 2002;88(5):2713-25.

33. Reinhart KM, Shuttleworth CW. Ketamine reduces deleterious consequences of spreading depolarizations. Exp Neurol. 2018;305:121-8.

34. Tang YT, Mendez JM, Theriot JJ, Sawant PM, Lopez-Valdes HE, Ju YS, et al. Minimum conditions for the induction of cortical spreading depression in brain slices. J Neurophysiol. 2014;112(10):2572-9.

35. Chebabo SR, Hester MA, Aitken PG, Somjen GG. Hypotonic exposure enhances synaptic transmission and triggers spreading depression in rat hippocampal tissue slices. Brain Res. 1995;695(2):203-16.

36. Goldlust EJ, Paczynski RP, He YY, Hsu CY, Goldberg MP. Automated measurement of infarct size with scanned images of triphenyltetrazolium chloride-stained rat brains. Stroke. 1996;27(9):1657-62.

37. Bederson JB, Pitts LH, Germano SM, Nishimura MC, Davis RL, Bartkowski HM. Evaluation of 2,3,5-triphenyltetrazolium chloride as a stain for detection and quantification of experimental cerebral infarction in rats. Stroke. 1986:17(6):1304-8.

38. Hatfield RH, Mendelow AD, Perry RH, Alvarez LM, Modha P. Triphenyltetrazolium chloride (TTC) as a marker for ischaemic changes in rat brain following permanent middle cerebral artery occlusion. Neuropathol Appl Neurobiol. 1991;17(1):61-7.

39. Juzekaeva E, Nasretdinov A, Gainutdinov A, Sintsov M, Mukhtarov M, Khazipov R. Preferential initiation and spread of anoxic depolarization in layer 4 of rat barrel cortex. Frontiers in cellular neuroscience. 2017;11:390.

40. Balestrino M, Somjen GG. Chlorpromazine protects brain tissue in hypoxia by delaying spreading depression-mediated calcium influx. Brain Res. 1986;385(2):219-26.

41. Hansen AJ, Zeuthen T. Extracellular ion concentrations during spreading depression and ischemia in the rat brain cortex. Acta Physiol Scand 1981;113(4):437-45.

42. Pietrobon D, Moskowitz MA. Chaos and commotion in the wake of cortical spreading depression and spreading depolarizations. Nat Rev Neurosci. 2014;15(6):379-93.

43. Harriott AM, Takizawa T, Chung DY, Chen SP. Spreading depression as a preclinical model of migraine. The journal of headache and pain. 2019;20(1):45

44. Ayata C, Shimizu-Sasamata M, Lo EH, Noebels JL, Moskowitz MA. Impaired neurotransmitter release and elevated threshold for cortical spreading depression in mice with mutations in the alpha1 A subunit of P/Q type calcium channels. Neuroscience. 2000;95(3):639-45.

45. Hertelendy P, Menyhart A, Makra P, Sule Z, Kiss T, Toth G, et al. Advancing age and ischemia elevate the electric threshold to elicit spreading depolarization in the cerebral cortex of young adult rats. J Cereb Blood Flow Metab. 2017;37(5):1763-75.

46. Jarvis CR, Anderson TR, Andrew RD. Anoxic depolarization mediates acute damage independent of glutamate in neocortical brain slices. Cereb Cortex. 2001;11(3):249-59.

47. Brisson CD, Andrew RD. A neuronal population in hypothalamus that dramatically resists acute ischemic injury compared to neocortex. J Neurophysiol. 2012;108(2):419-30.
48. Osehobo EP, Andrew RD. Osmotic effects upon the theta rhythm, a natural brain oscillation in the hippocampal slice. Exp Neurol. 1993:124(2):192-9.

49. Juzekaeva E, Gainutdinov A, Mukhtarov M, Khazipov R. Reappraisal of anoxic spreading depolarization as a terminal event during oxygenglucose deprivation in brain slices in vitro. Sci Rep. 2020;10(1):18970.

50. Brisson CD, Hsieh YT, Kim D, Jin AY, Andrew RD. Brainstem neurons survive the identical ischemic stress that kills higher neurons: insight to the persistent vegetative state. PLoS ONE. 2014:9(5):e96585.

51. Dreier JP, Reiffurth C. The stroke-migraine depolarization continuum. Neuron. 2015;86(4):902-22.

52. Esmann M, Fedosova NU, Marsh D. Osmotic stress and viscous retardation of the Na, K-ATPase ion pump. Biophys J. 2008;94(7):2767-76.

53. Kimelberg HK, Rutledge E, Goderie S, Charniga C. Astrocytic swelling due to hypotonic or high $\mathrm{K}+$ medium causes inhibition of glutamate and aspartate uptake and increases their release. J Cereb Blood Flow Metab. 1995;15(3):409-16.

54. Hertelendy P, Varga DP, Menyhart A, Bari F, Farkas E. Susceptibility of the cerebral cortex to spreading depolarization in neurological disease states: the impact of aging. Neurochem Int. 2019:127:125-36.

55. Lauderdale K, Murphy T, Tung T, Davila D, Binder DK, Fiacco TA. Osmotic edema rapidly increases neuronal excitability through activation of NMDA receptor-dependent slow inward currents in juvenile and adult hippocampus. ASN Neuro. 2015. https://doi.org/10.1177/1759091415 605115.

56. Anderson TR, Jarvis CR, Biedermann AJ, Molnar C, Andrew RD. Blocking the anoxic depolarization protects without functional compromise following simulated stroke in cortical brain slices. J Neurophysiol. 2005:93(2):963-79.

57. White $S H$, Brisson CD, Andrew RD. Examining protection from anoxic depolarization by the drugs dibucaine and carbetapentane using whole cell recording from CA1 neurons. J Neurophysiol. 2012;107(8):2083-95.

58. Richter F, Ebersberger A, Schaible HG. Blockade of voltage-gated calcium channels in rat inhibits repetitive cortical spreading depression. Neurosci Lett. 2002;334(2):123-6.

59. Szabo I, O MT, Torok Z, Varga DP, Menyhart A, Frank R, et al. The impact of dihydropyridine derivatives on the cerebral blood flow response to somatosensory stimulation and spreading depolarization. Br J Pharmacol. 2019;176(9):1222-34

60. Dijkhuizen RM, Beekwilder JP, van der Worp HB, Berkelbach van der Sprenkel JW, Tulleken KA, Nicolay K. Correlation between tissue depolarizations and damage in focal ischemic rat brain. Brain Res. 1999;840(1-2):194-205.

\section{Publisher's note}

Springer Nature remains neutral with regard to jurisdictional claims in published maps and institutional affiliations.

Ready to submit your research? Choose BMC and benefit from

- fast, convenient online submission

- thorough peer review by experienced researchers in your field

- rapid publication on acceptance

- support for research data, including large and complex data types

- gold Open Access which fosters wider collaboration and increased citations

- maximum visibility for your research: over 100M website views per year

At BMC, research is always in progress.

Learn more biomedcentral.com/submissions 